\title{
Rotavirus diarrhea - An analysis through epidemic modeling
}

\author{
Ojaswita Chaturvedi*1 ${ }^{* 1}$ Edward Lungu², Mandu Jeffrey ${ }^{3}$, Shedden Masupe ${ }^{4}$ \\ ${ }^{1}$ Udarta Marketing Pvt Ltd, Founder, Director-Research and Development, Pune, India \\ ${ }^{2}$ Botswana International University of Science and Technology, Gaborone, Botswana \\ ${ }^{3}$ Department of Electrical and Electronics Engineering University of Botswana, Gaborone, Botswana \\ ${ }^{4}$ Botswana Institute of Technology Research and Innovation, Gaborone, Botswana
}

Received: August 21, 2016

DOI: $10.5430 /$ jbei.v4n2p21
Accepted: December 28, $2018 \quad$ Online Published: December 30, 2018

URL: https://doi.org/10.5430/jbei.v4n2p21

\begin{abstract}
Modelling an infectious disease like diarrhea improves the understanding of the transmission and helps in preventing it. Based on the pathogenesis, this research creates a continuous mathematical model for diarrhea (forming an SIRS system) caused by the virus rotavirus. Basic calculations including the basic reproduction number $\left(R_{0}\right)$ and disease-free state are successfully completed for the described system. Results show that if the basic reproduction number is kept below 1 (i.e. $R_{0}<1$ ), there will be no epidemic. This mathematical model has been simulated using assumed values of parameters to test its fidelity. In order to establish the model in a functioning form, the Routh Hurwitz method has been used for the stability analysis. Model fidelity has been made stronger by conducting the sensitivity analysis and parameter estimation too. This model is very useful and has been integrated to form utilizable systems which function as a predictive system to assist in prevention of further infections of rotavirus causing diarrhea.
\end{abstract}

Key Words: Modeling, Epidemics, Mathematical mmodeling, Diarrhea, Rotavirus, Routh Hurwitz analysis, Sensitivity analsysis, Parameter estimation

\section{INTRODUCTION}

Human population at-large, is continuously affected by the threats presented by infectious diseases. The World Health Organisation (WHO) records that $43 \%$ of the total global disease burden is caused by infectious disease. ${ }^{[1]}$ Furthermore, it was established in 2008 that out of all deaths, $16 \%$ was due to infectious diseases. ${ }^{[2]}$ Generally, a closed population is exposed to Infectious disease through a carrier of infectious agents. A cariier can differ, varying from a patient or any infected person to any external vector like water, body fluids or air. Uncontrolled transmissions of such diseases results in an epidemic. Definition of an epidemic is given as a abrupt and sharp increment in the number of cases within a closed population. ${ }^{[3]}$ An increase in the number of cases allows transmission of the disease to be more instantaneous and in this way it increases the mortality rate of the population.

As it is essential to life, water related diseases form a significant portion of infectious diseases. One of the most popular diseases that is transmitted through water is diarrhea. Alarming global health statistics are produced by the disease reaching up to 1.7 billion cases annually as per WHO records. Pneumonia topping the list among causes of child mortality,

\footnotetext{
* Correspondence: Ojaswita Chaturvedi; Email: krishnaa.chaturvedi@ gmail.com; Address: Udarta Marketing Pvt Ltd Founder, Director - Research and Development Pune, India.
} 
diarrhea is the second. ${ }^{[4]}$ Diarrhea continues to threat even countries like Botswana that are land-locked. Principal water territorials around the country and the bulky habitations of population around them form an easy transmission pathway for diseases like diarrhea. Persons who fall within the lower socio-economic level are more likely to catch the disease due to lesser or no access to appropriate treatment and/or methods of prevention. When treatments are administered in such habitats at all, they are most likely adapted inadequately thus becoming unfruitful. The persistence of diarrheal diseases in Botswana despite provision of treatment methods such as rehydration methods like Oral Rehydration Salt (ORS) therapies is confirmed by a recent research. ${ }^{[5]}$ In this way, an easy derivation can be made that prevention strategies for diseases like diarrhea are urgently required despite the management of treatments in order to achieve a significant reduction in mortality rates and cases.

Diarrhea is a disease which can easily be transmitted as it can be activated and communicated by a number of genres among bacteria, virus and protozoa families. One of the most perilous pathogen in relation to diarrhea is the rotavirus. Rotavirus is classified into several serotypes which can cause viral gastroenteritis. Gastroenteritis is the inflammation of the gastrointestinal tract and has common symptoms of diarrhea, vomiting, fever and abdominal pains. ${ }^{[6]}$ Rotavirus is one of the foremost sources of diarrhea around the world and results in approximately 527,000 deaths annually. One of the most hazardous diseases causing about 900,000 deaths annually happens to be malaria. The figures produced by rotavirus can easily be compared to this mortality rate, hence proving the importance of the study and prevention of the disease. On the national level, Rotavirus remains the leading pathogen for diarrhea infections in Botswana too. ${ }^{\text {[7] }}$

Epidemic modelling is used to give a profound understanding regarding the dynamics of such diseases thus help in further analysis regarding prevention. Epidemic models are plainly tools that help in predicting infection process and expected outbreaks of the disease. Prevention is an important aspect as it can prove to be successful in combating diarrhea for the human population. A model, in the continuous mathematical format, for rotavirus diarrhea has been composed, analysed and simulated in this study. This model forms a section of an envisioned complete interface that is capable of visually assuring the presence or absence of an epidemic in a population and also presentating the variation of the population with respect to time in either of the cases.

It is foreseen that avoidance methods will be appreciably improved by the systems that are to come up by executing such epidemic models. Using the system described below, it will be possible to prevent the transmission to a noteworthy point. Diseases like rotavirus diarrhea not only present alarming mortality rates but also contribute towards massive financial burdens under the veil of misconception of less hazard and easy treatment. ${ }^{\left[{ }^{-11]}\right.}$ Therefore, the system being constructed will help the attenuation of monetary load as well. The system characterizes a set of easy-to-use features which do not require any intense scientific or technological knowledge for operation. Hence, the proposed system is envisaged to minimize the hazards of rotavirus.

\section{BACKGROUND}

\subsection{Epidemic modeling theory}

Epidemic models came into the research world as early as 1927 when Karmack and McKendrick formulated the first epidemic compartmental model. ${ }^{[12]}$ Three compartments the susceptible, infected and recovered combined together form this model and hence it is known as the SIR model. Figure 1 shows the illustration of the basic SIR model as formulated in 1927. Differential equations are used to describe the compartments of the model, explaining the variations that take place in the class with respect to time. Figure 1 shows the model in a simple diagrammatic form.

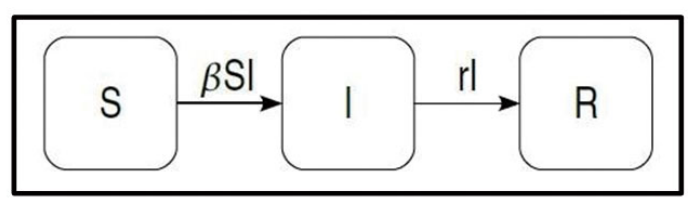

Figure 1. SIR model

Complete definition of the epidemic model is done using ordinary differential equations (ODEs) along with a number of pre-defined parameters. The basic SIR model comprises of two parameters which are: $\beta$ - the infectivity rate and the recovery rate is denoted as $r$.

$$
\begin{aligned}
& \frac{d \mathrm{~S}}{d t}=-\beta S I \\
& \frac{d \mathrm{I}}{d t}=\beta S I \\
& \frac{d \mathrm{R}}{d t}=r I
\end{aligned}
$$

Mathematically, the differential equations are used to depict any changes that arise in each class wrt time. For any epidemic model, the entire set of compartments is related 
to each other which is shown by the inculcation of population classes within the differential equations. Through these equations, it becomes possible to quantitatively observe and analyze the changes occurring in the population of every compartment and in addition examine the movement of the population from one compartment to another.

The basic SIR epidemic model has been used to formulate numerous other models for infectious diseases. Being vast themselves, infectious diseases have been successfully represented and investigated using epidemic modeling and mathematical models by research centres all around the world. Diseases like malaria, HIV infections, hepatitis and influenza have been modeled mathematically giving important end results. ${ }^{[13-15]}$

\subsection{Pathogenesis and epidemiology of rotavirus diar- rhea}

Epidemic modelling is an effective effort that can be customised to prevent the outbreaks of infectious diseases. Out of the infectious water-borne diseases, diarrhea occupies a hazardous position endangering human life across a significant percentage. Diarrhea can be caused by bacteria, virus or parasitic organisms that are transferred through the human population via unhygienic food and water. For effective modelling, it is compulsory to understand the characteristics of the disease and the pathogens causing it.

Diarrhea is a disease which has the key symptom of passing of two or more liquid stools in a day, causing dehydration. Fever and abdominal cramps are other symptoms associated with the disease. ${ }^{[16]}$ Significant amounts of liquid loss together with other important salts/minerals/chemicals results due to dehydration. Such situations can be tremendously callous with specific connotation in smaller children and can even result in death if not controlled within time. Although bacterial causes of the disease form the major disease load, Rotavirus is also found out to be on the top of the list. ${ }^{[17]}$

Various pathogens cause diarrhea and it appears in the human body in two ways namely as a symptom of another disease or as a disease itself. As per the Botswana national records, there are four major pathogens that are listed as the topmost carriers of diarrhea in the country: Rotavirus among the viri, shigella and salmonella from the bacterial causes and cryptosporidium among the protozoa. Rotavirus is found to occupy the highest position in the above list.

The recorded infective dose of rotavirus is 10-100 viral particles $^{[18]}$ and trillions of virions are released from an infected individual during one diarrheal episode. ${ }^{[19,20]}$ Rotavirus diarrhea has an incubation period from two to three days. For the duration of the incubation period, the patient does not

Published by Sciedu Press show any symptoms but due to its elevated levels of transmission capabilities and contagiousness, the virus (rotavirus) is transmitted into the environment during this period too. Transmission of rotavirus occurs through contaminated food, water or any item that comes in contact with polluted hands, e.g. toys. A person infected with rotavirus is highly infectious or contagious for up to ten days after the symptoms begin and he/she may continue to spread the virus after this period but at considerable slower. ${ }^{[18]}$ Rehydration is the main treatment given to the infected patients of (rotavirus) diarrhea. As of date, there are no particular medicines that can be used to treat the rotavirus. The mainstay of treatment is supportive through oral rehydration.

With the implement of the rotavirus vaccine, there are many ongoing research works that elaborate models including vaccination strategies. ${ }^{[21,22]}$ Simple rotavirus models have also been researched on with respect to rodents. ${ }^{[23,24]}$ Considering the fact that rotavirus vaccines are in the introductory phase and still not in abundance in rural areas, ${ }^{[25,26]}$ it is important to have a working model that describes the straight dynamics of the pathogen so that the combat methods that are built using the model are more realistic.

\section{MODEL DEVELOPMENT}

\subsection{Description}

The basic SIR model has been elaborated with a few extensions to fully describe the rotavirus dynamics. Depending on the pathogenesis, the model has been built into four compartments, which are each detailed as given below:

(1) Susceptible (S) - The susceptible class is the section of individuals from the entire population that are vulnerable to the disease

(2) Asymptomatic Infected (IA) - This is the group of people who have the pathogen within them but so not show any symptoms of the disease, yet they continue to contribute to the pathogen population

(3) Symptomatic Infected (IS) - Symptomatic infected are the persons who have ingested the pathogen and have begun to demonstrate symptoms of the disease. This group also contributes to the pathogen population

(4) Recovered ( R) - The recovered individuals are those who have totally recovered from the disease and cannot spread it further

Figure 2 shows a diagramatic representation of the Rotavirus model. All the associated parameters of the model are listed and described in Table 1. The values of these parameters will only be used at a later stage. These values have been assumed since calculations were not possible due to the unavailability of data. Therefore they do not necessarily convey a practical 
situation wholly at this stage but are good enough to outline the scenario of an epidemic.

Table 1. Parameter Descriptions

\begin{tabular}{lll}
\hline Parameter & Description & Value \\
\hline$\beta$ & Infectivity Rate & \\
$\alpha$ & Rate of immunity loss & 0.25 \\
$\mu$ & Natural death rate & 0.012 \\
$\mathrm{p}$ & Asymptomatic infection probability & 0.86 \\
$\mathrm{r}$ & Rate of recovery & 0.8 \\
$\delta(=1-\mathrm{p})$ & Of symptomatic infection probability & 0.14 \\
$\mathrm{k}$ & Rate of Symptom gaining & 0.25 \\
$\Pi$ & Population renewal rate & 22 \\
\hline
\end{tabular}

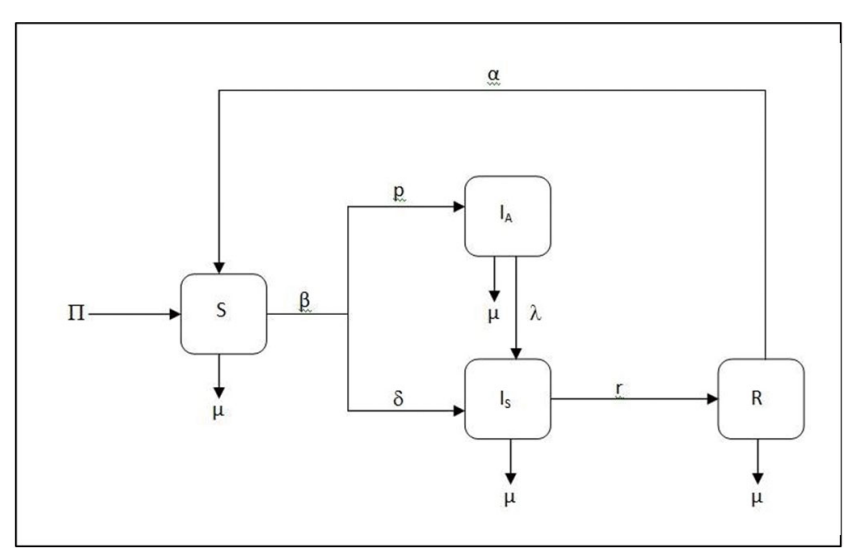

Figure 2. Schematic Representation of Model

In order to complete the initial stages of simulation, parameters values were either assumed or analyzed using certain literatures sources. Taking an assumed population of approximately 2,000 and a growth rate of $1.1 \%$, the population renewal has been calculated as 22 . Considering a timeframe of 15 days for the experimental simulation and the approximate time taken for rotavirus symptoms to manifest to be 3 to 4 days, the rate of gaining symptoms is taken to be 0.25 . As rehydration is the primary treatment option, the recovery rate is largely dependent on the availability of clean water (which ensures total flush out of infecting pathogens from the patient's body lest he remain a carrier), the direct recovery rate is taken to be 0.8 which has been derived from the fact that approximately $80 \%$ of the population in Botswana gets piped water.

From the susceptible persons group. A particular fraction can become infected to either directly start showing symptoms (symptomatic) or become asymptomatic infectious. Two of the infectious classes contribute to the infection growth at various rates. After a specific period of time, according to the dose of the virus in the body, the asymptomatic individuals become symptomatic. Infectious individuals gain recovery with the aid of body immunity, medication and/or rehydra- tion therapies. The human body preserves an immunity level for a certain time period but eventually, this level declines and the recovered person becomes a susceptible individual who can catch the disease.

\subsection{Assumptions considered for the modeling}

Any modelling processes cannot be completed without assumptions. For this process, the following assumptions have been used:

(1) All individuals in the population are equally susceptible

(2) Disease induced mortality is negligible under the temporal scale consideration therefore no individual dies due to infection

(3) No cases of co-infection are involved

(4) No cases of multiple pathogen infection are measured

(5) All individuals that are born in the population are susceptible to the disease

(6) Both direct and indirect infection are combined for infections described in the model

(7) The population is well mixed i.e. the infected people are homogeneously spread throughout the entire population

By using the above described parameters and assumptions, the extended compartmental model can be defined mathematically using the following differential equations:

$$
\frac{d \mathrm{~S}}{d t}=\Pi-\mu S-\beta S\left(p_{A}+\delta_{S}\right)+\alpha R
$$

Equation 4 describes the how the susceptible class changes with respect to time. New individuals are added in this class through a constant recruitment/birth rate $\pi$ runs and constant death rate $\mu$ decreases this class. A percentage of the susceptible get infected depending on an infectivity rate $\beta$. Recovered individuals slowly seam into the susceptible class depending on an immunity loss rate $\alpha$.

$$
\frac{d I_{A}}{d t}=\beta p S I_{A}-\mu I_{A}-k I_{A}
$$

Equation 5 describes the change in the asymptomatic infectious population. A portion of the susceptible class at becomes asymptomatic infectious depending on the infectivity rate $\beta$ and probability $p$. The above class gets reduced by a constant death rate $\mu$ and symptom gain rate $k$.

$$
\frac{d I_{S}}{d t}=\beta \delta S I_{S}-\mu I_{S}-r I_{S}+k I_{A}
$$


The above equation defines the symptomatic infectious population. This compartment is fed in by the susceptible population that becomes symptomatic at an infectivity rate $\beta$ and probability $\delta$. A constant death rate $\mu$ reduces the symptomatic population and a certain percentage of the class becomes recovered at a recovery rate $\mathrm{r}$.

$$
\frac{d R}{d t}=r I-\mu R-\alpha R
$$

The recovered class of the model is described through equation 7. This class gets an increment in population as the symptomatic population recovers at the recovery rate $\mathrm{r}$. A constant death rate $\mu$ reduces population from the recovered class. Immunity is lost at rate $\alpha$ and these individuals move into the susceptible class.

\section{Model ANALYSiS}

\subsection{Calculation of the disease free equilibrium and basic reproduction number}

The Disease-free equilibrium or Virus-free equilibrium (VFE) is the no infection state of the population i.e. where there are no infectious people in the population. For the population to be infection-less, all the infected states will be assumed to be zero.

No infectious population directly implies that the recovered population is also 0 . Therefore $\mathrm{R}=0$. In conclusion, the only non-zero class at the VFE is the susceptible class. At the VFE, all the classes will be indicated with an asterisk.

In order to get the asymptotic state, the right hand side of equation 4 should be equated to zero.

$$
\begin{aligned}
& \Pi-\mu S^{* *}=0 \\
& S^{* *}=\frac{\pi}{\mu}
\end{aligned}
$$

Thus, the VFE is defined as follows

$$
V F E=(\stackrel{11}{-}, 0,0,0)
$$

Firstly, for the above calculation, the classes that contain new infection parameters need to be considered. The susceptible and recovered classes are not related to new infections, thus, the Asymptomatic infectious and the Symptomatic infectious classes will be taken into consideration for the calculation of the basic reproduction number.

The new infections in the three classes mentioned above can be denoted in the matrix below, using equations (2) and (3).

$$
\mathcal{F}=\left[\begin{array}{l}
\beta p S I_{A} \\
\beta \delta S I_{S}
\end{array}\right]
$$

The other changes in the same classes can also be shown in a matrix. Note that the signs of the respective terms will change in this case.

$$
\mathbb{V}=\left[\begin{array}{l}
(\mu+\lambda) I_{A} \\
(\mu+r) I_{S}
\end{array}\right]
$$

Calculating the Jacobian Matrix of each of the above matrices, we get:

$$
\begin{gathered}
F=\left[\begin{array}{cc}
\beta p S & 0 \\
0 & \beta \delta S
\end{array}\right] \\
V=\left[\begin{array}{cc}
(\mu+\lambda) & 0 \\
0 & (\mu+r)
\end{array}\right]
\end{gathered}
$$

At the VFE, the replacement $S^{* *}=\frac{\Pi}{\mu}$ can be used and the above matrices can be written as:

$$
F=\left[\begin{array}{cc}
\frac{\beta p \Pi}{\mu} & 0 \\
0 & \frac{\beta \delta \Pi}{\mu}
\end{array}\right]
$$

The basic reproduction number can be calculated using the Jacobian matrix and next generation method. The expression of $R_{0}$ is found out to be:

$$
R_{0}=\frac{\beta p \Pi}{\mu(\mu+\lambda)}
$$

\subsection{Results - Simulation and discussion}

Primary simulations were done of the continuous model in order to ascertain the fidelity, stability and workability of the model prior to the compound analysis. Owing to the unavailability of data specific to pathogens in diarrhea, the parameters used for the first simulation were assumed/analyzed as described in Section 3.1.

The value of $R_{0}$ - the basic reproduction number is reliant on the infectivity rate. If the basic reproduction number is one, a value of the infectivity rate is calculated and used to maneuver whether an epidemic is present or not in the population. Excepting the death rate, the remaining parameters have been designated values which are listed in Table 1. For this study, we use the infectivity rate $\beta$ to control the value of the basic reproduction number $-R_{0}$. We use the derived 
definition of $R_{0}$ and equate to 1 to obtain an expression for $\beta$.

For $R_{0}=1$,

$$
\begin{gathered}
\beta p \Pi=\mu(\mu+\lambda) \\
=\frac{\mu(\mu+\lambda)}{\beta p \perp 1}=\frac{0.012(0.012+0.25)}{0.9 \times 22}=0.000159
\end{gathered}
$$

It can be followed that when $\beta<0.000159 ; R_{0}<1$ and there is no epidemic in the population. Similarly when $\beta>$ $0.000159 ; R_{0}>1$ and the presence of an epidemic is indicated in the population. The following simulations of the model show the changes in the compartments of the population for different values of $\beta$.
Figures 3 and 4 show the variations of the population for different infectivity rates. Figures $3 \mathrm{a}$ and $3 \mathrm{~b}$ show a nonepidemic situation as the values of $\beta$ are less than 0.000159 . It can be seen from the graphs that the susceptible population grows while the other compartments show a decline. In case of no epidemic, the susceptible population is not infected, thus it displays an increment. As no individuals are being fed into the infected classes from the susceptible class (due to low infectivity rate and no epidemic), the infected classes decrease in number. Following this, it is obvious that if there are no infected persons in the population, there will be no recovered people too. Thus the other classes show a decline. It can be deduced that as long as the value of $R_{0}$ is kept to be lower than 1 , there will be no epidemic, regardless of the exact value.

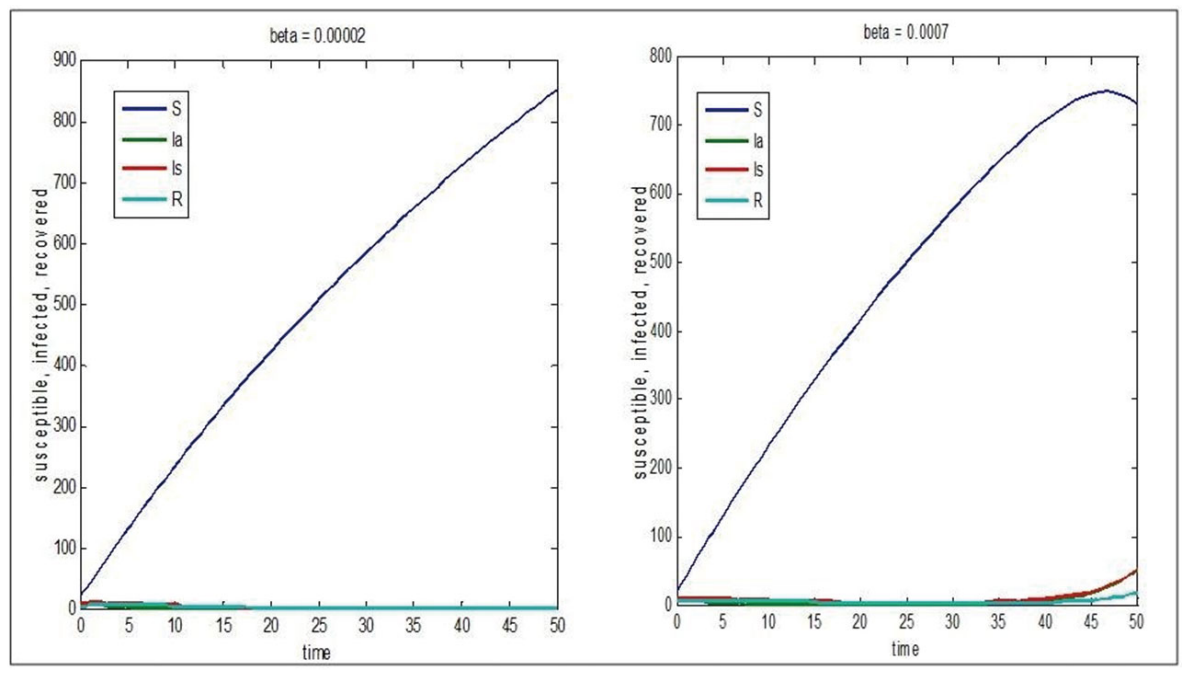

Figure 3. (a \& b) Rotavirus Simulations for $R_{0}<1$

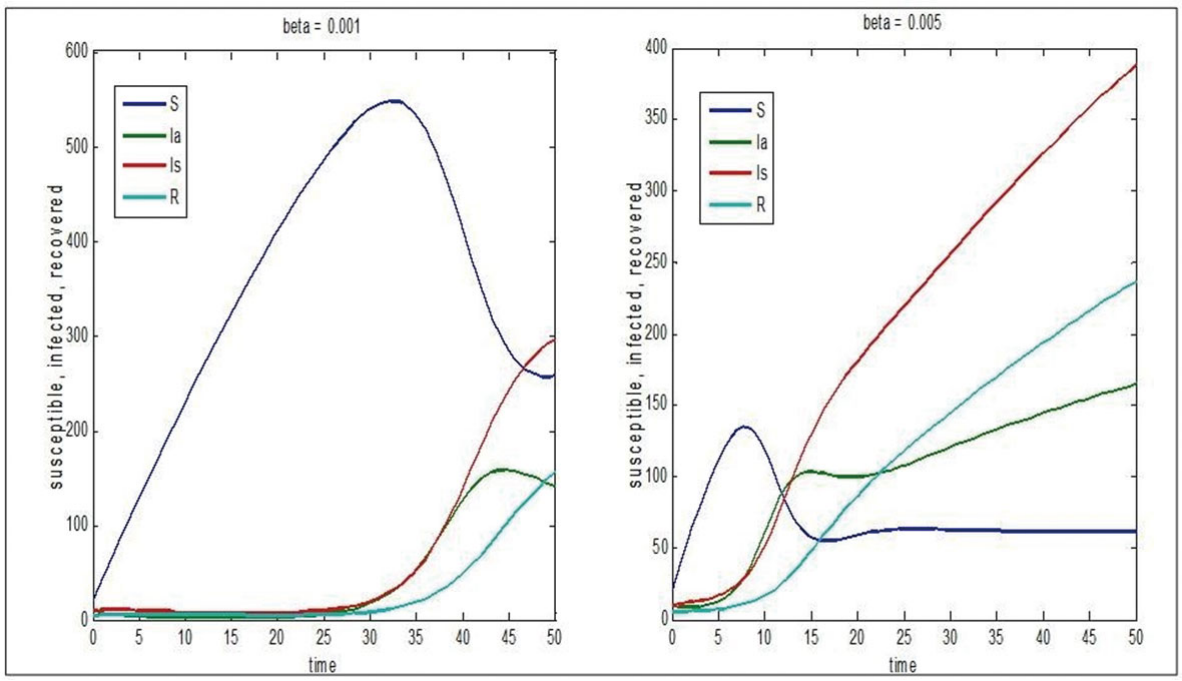

Figure 4. (a \& b) Rotavirus Simulations for $R_{0}>1$ 
Slightly increasing the infectivity rate introduces an epidemic in the population and the susceptible population begins to reduce as people get infected. This is shown in Figure 4a. Since the value of $R_{0}$ is increased by a small amount, the decline in the susceptible class only begins after a certain period of time. Because the susceptible class feeds into the infected classes, it reduces while the infected classes increase. As a result, the recovered population also increases. As the infectivity rate is increased, the value of $R_{0}$ also increases, thus presenting higher levels of epidemic in the population. This can be depicted in Figure 4. It can be seen from the figures that as the basic reproduction number increases, the infection spreads at higher rates, thus causing the susceptible class to show a decline at faster rates and reduce to lower values. For $\beta=0.0007$, the decline begins at 45 -time intervals, as the value of $\beta$ is increased to 0.001 , the decline in the susceptible population begins at 35-time intervals and there after the population reduces to significant levels (as depicted in Figure 4b). As the infectivity rate increases, the infected populations increase too. If the value of $R_{0}$ increases to higher values, the susceptible population immediately decreases and gradually diminishes. Figures 3 and 4 display this.

\subsection{Stability analysis}

One of the best methods to emasure stability is the Routh Hurwitz stability test which observe the system by make certain that all the roots of a specified polynomial are in the left half pane of the axis i.e. negative values (real or imaginary). To apply the theorem to any physical system, equations are used to describe the variations within. In epidemic modelling, population movement in between presecribed compartments is defined using ODEs. Stability of the solutions of these equations can be established using the Routh Hurwitz method.

Theorem 1. Upon solving, if the roots have negative values, the system will be stable.

In order to determine the stability of the rotavirus epidemic model, the initial step will be to consider the equation set that describe the model. In this step we presume that the rate of immunity loss, $\alpha$ is approximated to be 0 , for easing the calculation purposes.

$$
\begin{gathered}
f 1=\Pi-\mu S-\beta S\left(p I_{A}+\delta I_{S}\right) \\
f 2=\beta p S I_{A}-\mu I_{A}-l I_{A} \\
f 3=\beta \delta S I_{S}-\mu I_{S}-r I_{S}+l I_{A} \\
f 4=r I_{S}-\mu R
\end{gathered}
$$

Working out the Jacobian matrix of the above system, we get:

$$
J=\left[\begin{array}{cccc}
-\mu-\beta\left(p I_{A}+\delta I_{S}\right) & -\beta \delta S & -\beta p S & 0 \\
\beta \delta I_{S} & \beta S-\mu-r & k & 0 \\
\beta p I_{A} & 0 & \beta S-\mu-\lambda & 0 \\
0 & r & 0 & -\mu
\end{array}\right]
$$

The above Jacobian matrix will be substituted with the DFE expression for rotavirus to work out the stability. Doing this we get,

$$
\begin{gathered}
E_{0}=\left(\frac{\Pi}{\mu}, 0,0,0\right) \\
J\left(E_{0}\right)=\left[\begin{array}{cccc}
-\mu & \frac{-\beta \Pi \delta}{\mu} & \frac{-\beta \Pi p}{\mu} & 0 \\
0 & -\mu-r_{\beta} & k & 0 \\
0 & 0 & \frac{\beta}{\mu}-\mu-\lambda & 0 \\
0 & r & 0 & -\mu
\end{array}\right]
\end{gathered}
$$

When we subtract the index matrix multiplied by the operating parameter $\gamma$, we get:

$$
J\left(E_{0}\right)-\gamma=\left[\begin{array}{cccc}
-\mu-\gamma & \frac{-\beta \Pi \delta}{\mu} & \frac{-\beta \Pi p}{\mu} & 0 \\
0 & \frac{\beta \Pi}{\mu}-\mu-r-\gamma \beta \Pi & k & 0 \\
0 & 0 & \frac{\beta}{\mu}-\mu-k-\gamma & 0 \\
0 & r & 0 & -\mu-\gamma
\end{array}\right]
$$

The characteristic equation is derived using this matrix which is defined as:

$$
E_{\lambda}=(-\mu-\gamma) \times(-\mu-\gamma) \times\left(\frac{\beta \Pi}{\mu}-\mu-r-\gamma\right) \times\left(\frac{\beta \Pi}{\mu}-\mu-\lambda-\gamma\right)
$$

Calculations confirm roots of this equation to be:

$$
\begin{aligned}
& \gamma_{1}=-\mu \\
& \gamma_{2}=-\mu \\
& \gamma_{3}=\frac{\beta \Pi}{\mu}-\mu-r=R_{0}\left(\frac{\mu+k}{\Pi}\right)-\mu-r
\end{aligned}
$$

Following theorem 1, the results deduced are describe as: The VFE of the rotavirus system is locally asymptotically stable when $R_{0}<1$ and is instable if $R_{0}>1$. 


\subsection{Sensitivity analysis and parameter estimation}

Sensitivity analysis can be carried out using different methods and for this project the PRCC (Partial Rank Correlation Coefficient) method is implemented. The PRCC method has been determined as one of the most reliable methods for measuring monotonicity between actual data and model outputs in non-linear systems. ${ }^{[27,28]}$ This method is based on sampling and the Latin Hypercube Sampling (LHS) method (a type of Monte Carlo Sampling) is the most efficient procedures for sampling ${ }^{[29]}$ as it compactly stratifies the input parameters. PRCC assesses the potency between the inputs and outputs of the model using correlation using the sampling done by the LHS method. ${ }^{[30]}$ This method has been used as a Real system measurement whereby we compare the output of the system with a real set of data. ${ }^{[31]}$

Beacsue the package for sensitivity analysis was accessible in MATLAB, the process became easier to execute as manual manipulations were cut down. Sensitivity analysis has been done with respect to the basic reproduction number parameter for the rotavirus model i.e. the process involved the determination of the parameters that cause a major alteration in the basic reproduction number, thus affecting the epidemic state of a population. This conclusion was derived as the basic reproduction number is the determinant point of the epidemic in epidemiological studies - which means that absence or presence of an epidemic is completely dependent on the basic reproduction number. Once the results were obtained, the parameter values that resulted from the analysis were used for complex estimation so that the model simulations are in monotone with the real data available.

Sensitivity analysis is measured using the sensitivity index. A sensitivity index of 1 indicates a 50\% variation in the final value and the indices lie between -1 and 1 . Indications between -1 and 0 show a negative fluctuation while those between 0 and 1 show a positive variation.

The Figure 5 demonstrates the sensitivity analysis result for the rotavirus model. From Figure 5, it is clear that the infectivity rate and the probability of asymptomatic infection affect the basic reproduction number of the rotavirus model to significant levels. Based on the sensitivity index value of these parameters (which is approximately 0.65 and 0.68 ), it can be deduced that there will be a change of approximately $35 \%$ from the actual values. The symptom gaining rate also has an effect on the final value. Although this is a large value too, the other two parameters cause a threefold change and therefore, during the parameter estimation procedures, it is these values that will be altered such that the model gives appropriate results on simulation.

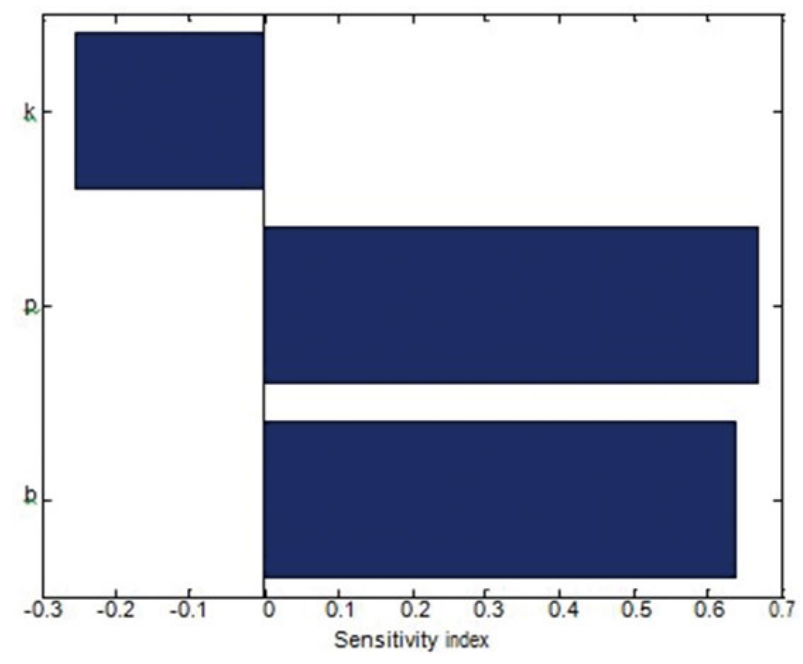

Figure 5. Sensitivity Analysis for Rotavirus Model

After completing the sensitivity analysis, parameter estimation is done. Parameters causing significant changes are chosen for alteration in this technique. Graphs are plotted for actual data and simulation results. The appropriate parameters are altered until the two graphs are in monotone with each other.

Since data was unavailable in Botswana for the specific pathogens, literature was used to get data from other locations. ${ }^{[32]}$ Since the rotavirus infectivity is highly affected by temperatures, the seasons were correlated for both countries and results were represented for Botswana as shown in Table 2. Infectivity rate has been designed to be dependent on the seasons therefore the calendar of the locations had to be matched with Botswana seasons before the matching of data and simulation was done.

Table 2. Rotavirus Interpreted Data for Botswana

\begin{tabular}{ll}
\hline Month & No. of cases \\
\hline January & 40 \\
February & 30 \\
March & 30 \\
April & 150 \\
May & 305 \\
June & 700 \\
July & 498 \\
August & 350 \\
September & 235 \\
October & 50 \\
November & 60 \\
December & 50 \\
\hline
\end{tabular}

Table 3 shows the values estimated for the rotavirus model. The matching of actual data with simulations is shown subsequently. Figure 6 shows the data fitting curves which 
was obtained by altering parameter values to gain maximum monotone between the original data and simulation produced numbers.

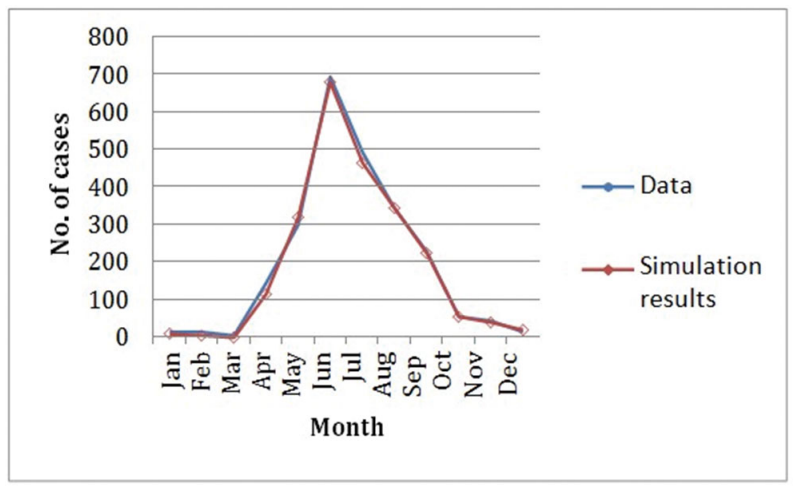

Figure 6. Data Fitting for Rotavirus Model Parameter Estimation

\section{Conclusion}

Epidemic modeling has been used in this study to understand the dynamics of rotavirus diarrhea. The basic reproduction number, $R_{0}$, was used to determine the existence of an epidemic. Routh Hurwitz method was used to carry out the stability analysis and the PRCC method was successfully used for sensitivity analysis. Sensitivity analysis gave the results for parameter estimation. Population variation was successfully obtained in the context of the pathogen in different seasons. This model is now ready to be integrated onto a complex and user friendly interface that will help in preventing further infection.

Table 3. Estimated Parameters for Rotavirus Model

\begin{tabular}{ll}
\hline Parameter & Value \\
\hline$\alpha$ & 0.5 \\
$\mathrm{p}$ & 0.2 \\
$\mathrm{r}$ & 0.6 \\
$\delta$ & 0.8 \\
\hline
\end{tabular}

\begin{tabular}{lll}
\hline Season & Description & 及 value \\
\hline April-May & Winter Approaching & 0.000009 \\
July- September & Summer Approaching & 0.0000005 \\
October- March & Dry Season & 0.0000004 \\
\hline
\end{tabular}

\section{REFERENCES}

[1] Global burden of disease, WHO programmes and projects. Available from: www . who. int. Accessed on 31/8/13

[2] Bayor College of Medicine, Department of Molecular Virology and Microbiology, Research - Emerging Infectious Diseases.

[3] Dicker RC, Coronado F, Koo D, et al. Principles of Epidemiology in Public Health Science, Third edition, Centers for Disease Control and Prevention (CDC).

[4] Diarrhea Fact Sheet, WHO Media centre. Available from: www. who. int. Accessed on 31/8/13.

[5] Jammalamadugu SB, Mosime B, Masupe T, et al. Assessment of the household availability of oral rehydration salt in rural Botswana. Pan African Medical Journal. 2013.

[6] Centers for Disease Control and Prevention, Division of Viral Diseases. Available from: www.cdc.gov/ncidod/dvrd/revb/gastr o/faq.htm. Retrieved om 21 November 2012

[7] Basu G, Rossouw J, Sebunya TK, et al. Prevelance of Rotavirus, Adenovirus and Astrovirus infection in young children in Botswana. East African Medical Journal. 2003; 80.

[8] Latipov R, Kuatbaeva A, Kristiansen O, et al. Economic burden of rotavirus disease in children under 5 years in Kazakhstan. Vaccine. 2011; 29.

[9] Rheingans RD, Antil L, Dreibelbis R, et al. Economic costs of Rotavirus gastroenteritis and cost effectiveness of vaccination in developing countries. International Journal of Infectious Diseases. 2009; 200 .

[10] Atherly DE, Lewis KD, Tate J, et al. Projected health and economic impact of rotavirus vaccination in GAVI-eligible countries: 20112030. Vaccine. 2012; 14.

Published by Sciedu Press
[11] Standaert B, Harlin O, Desselberger U. The Financial Burden of Rotavirus Disease in Four Countries of the European Union. The Paediatric Infectious Disease Journal. 2007; 12.

[12] Kermack WO, McKendrick AG. A contribution to the mathematical theory of epidemics. Proceedings of the Royal Society of London. $1927 ; 115$.

[13] Coburn BJ, Wanger BG, Blower S. Modeling influenza epidemics and pandemics: insights into the future of swine flu (H1N1). Biomedical Modelling Center. 2009; 7.

[14] The Kirby Institute for infection and immunity in the society. Available from: http://www.kirby. unsw. edu.au/

[15] Jian-quan L, Jie L, Mei-zhi L. Some discrete SI and SIS models. Applied Mathematics and Mechanics. 2012; 29.

[16] Cianflone NFC. Salmonellosis and the GI tract: More than just peanut butter. Current Gastroenetrology Reports. 2008; 10.

[17] Farthing M, Lindberg G, Dite P, et al. Acute Diarrhea, World gastroenterology Organisation Practice Guideline. 2008.

[18] Clark S, Graz M. Rotavirus. Available from: www . waterbornepa thogens . org. Retrieved on 27 March 2013.

[19] Bayor College of Medicine, Department of Molecular Virology and Microbiology. Research - Emerging Infectious Diseases.

[20] Walsh M. Rotavirus, Infection Landscapes. Available from: http:// www.infectionlandscapes.org/2011/11/rotavirus.html. Retrieved on 22 December 2015.

[21] Ortega OY. Evaluation of rotavirus models with coinfection and vaccination. Thesis Abstract, University of Iowa. 2008.

[22] Pitzer VE, Atkins KE, Freiesleben de Blasio B, et al. Direct and Indirect Effects of Rotavirus Vaccination: Comparing Predictions from Transmission Dynamic Models. PLoS ONE. 2012; 7(8): e42320.

[23] Knipping K, Mcneal MM, Crienen A, et al. A gastrointestinal rotavirus infection mouse model for immune modulation studies. Virol- 
ogy Journal. 2011; 8: 109. PMid:21385425. https ://doi .org/10 $.1186 / 1743-422 \mathrm{X}-8-109$

[24] Perez Cano FJ, Castell M, Castellote C, et al. Characterization of Clinical and Immune Response in a Rotavirus Diarrhea Model in Suckling Lewis Rats. Pediatric Research. 2007; 62: 658-663. PMid:17957154. https://doi.org/10.1203/PDR.0b013e318159a273

[25] Gastanaduy PA, Steenhoff AP, Mokomane M, et al. Effectiveness of Monovalent Rotavirus Vaccine After Programmatic Implementation in Botswana: A Multisite Prospective Case-Control Study. Clinical Infectious Diseases. 2016; 62(S2): S161-7.

[26] MOH - UNICEF, WHO, 2013 Botswana: Post Introduction Evaluation of Pneumococcal and Rotarix Vaccines in Botswana, Unicef Evaluation Database.

[27] Marino S, Hogue IB, Ray CJ, et al. A Methodology For Performing Global Uncertainty And Sensitivity Analysis In Systems Biology.
Journal of Theoretical Biology. 2008; 254: 178-196. PMid:18572196. https://doi.org/10.1016/j.jtbi.2008.04.011

[28] Hamby DM. A Comparison Of Sensitivity Analysis Techniques. Westinghouse Savannah River Company.

[29] Helton JC, Davis FJ, Johnson JD. A comparison of uncertainty and sensitivity analysis results obtained with random and Latin hypercube sampling. Reliability Engineering \& System Safety. 2005; 89.

[30] Thiele JC, Kurth W, Grimm V. Facilitating Parameter Estimation and Sensitivity Analysis of Agent-Based Models: A Cookbook Using NetLogo and R. Journal of Artificial Societies and Social Simulation. 2014; 17.

[31] David J. Testing and Validation of Computer Simulation Models: Principles, Methods and Applications, Springer; 2015.

[32] Health Protection Surveillance Centre, Ireland. Available from: www.hpsc.ie 